\title{
Laboratory Database to Manage Electrophoresis and Chromatography Separations and the Associated Samples
}

BioTechniques 29:1256-1262 (December 2000)

\author{
Kenneth D. Cole \\ National Institute of Standards \\ and Technology, Gaithersburg, \\ MD, USA
}

\section{INTRODUCTION}

Two significant challenges in a biotechnology laboratory are the management of large numbers of samples and the data resulting from the analysis of these samples. Separation technologies are used in virtually all biotechnology laboratories to monitor reactions, to characterize complex samples, and to isolate products. Preparative separations produce a large number of fractions that often must be analyzed by using analytical separation techniques.

In our laboratory, we analyze a large number of samples using gel electrophoresis to determine the performance of preparative chromatography techniques (and other techniques such as membrane separations). To determine the efficiency of a separation, we use mixtures of DNA (or proteins) as test samples. Through repeated use and analysis, these mixtures become laboratory standards, and their analytical and preparative separation histories increase their value.

In the past, we have used forms to help us record all the necessary data on a separation technique and the associated samples. We decided that a well-designed database would facilitate the collection, organization, and retrieval of this data. Modern relational databases are very powerful in their ability to store and retrieve large amounts of data. A database (based on FileMaker Pro $\left.^{\circledR}\right)$ has been recently developed that manages a large amount of information on biological samples, protocols, order- ing information, and other data for a biotechnology laboratory (2). Relation databases for the management of laboratory data have become increasing popular (3). A properly designed database will prompt a researcher to collect all the necessary data and will store it in a form that will allow easy retrieval when needed.

Gel electrophoresis and column chromatography are very mature techniques with hundreds of applications. Individual laboratories routinely establish their own conditions that work best for their particular samples. This database will assist researchers in storing the separation conditions and will allow the rapid and easy query of large amounts of historical data from the separation and characterization of complex stocks, fractions, and standards.

The Microsoft ${ }^{\circledR}$ Office Suite is becoming increasingly popular and includes a powerful database (Microsoft Access $\left.{ }^{\circledR}\right)$ that is suitable for small to medium size applications. We have developed a laboratory separation database using Access because of its popularity and compatibility with other Office programs.

\section{MATERIALS AND METHODS}

The separation database was developed using Access 97. The program will run on the Microsoft Windows ${ }^{\mathrm{TM}}$ $(95,97$, or NT) operating systems. The database without data occupies approximately $0.7 \mathrm{MB}$. 
Relationships for NewSep2000

Thursday, June 22, 2000

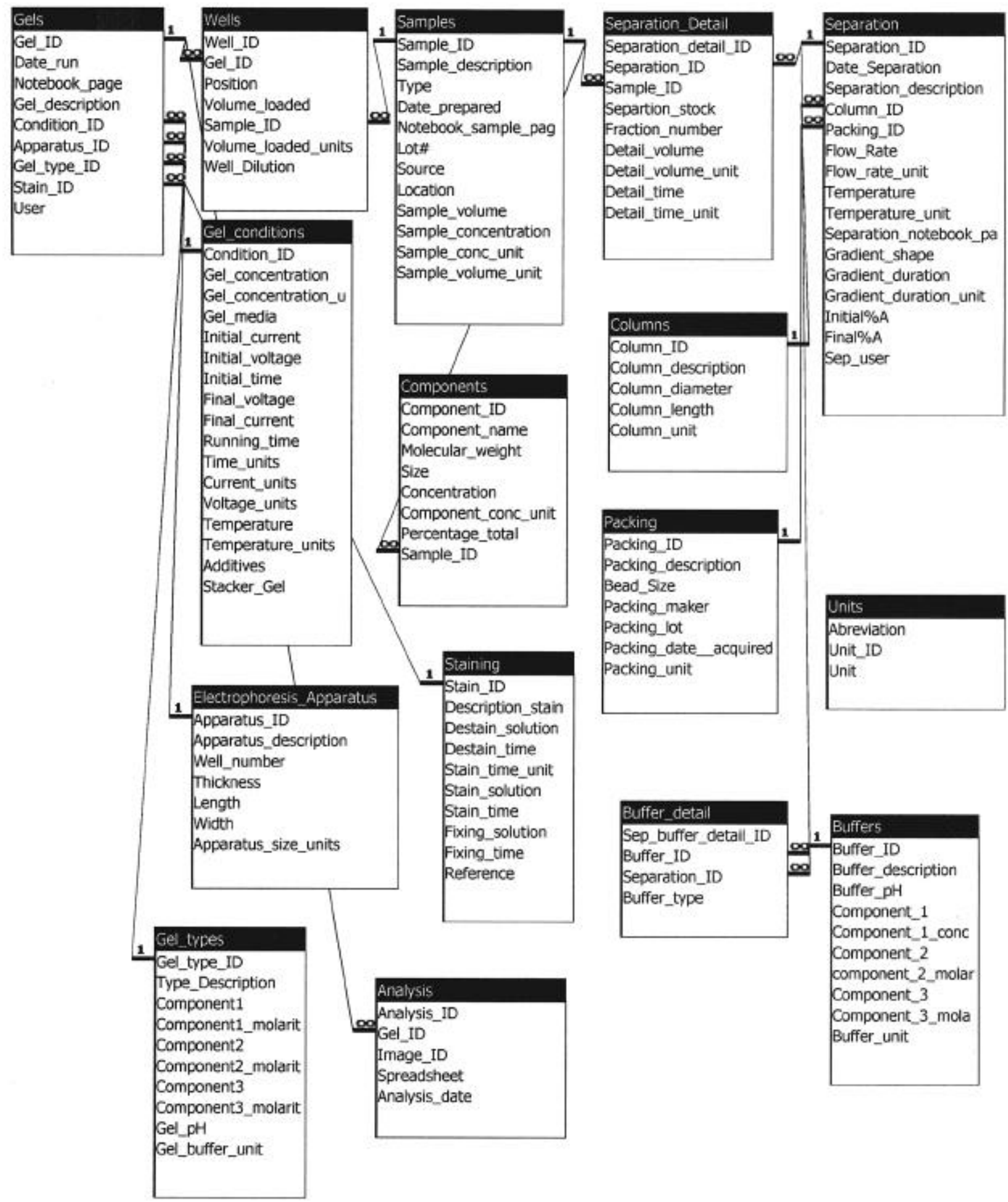

Figure 1. Tables and relationships for the separations database. Table names are on top, and data attributes are shown below. Relationships are shown by connecting lines from the primary keys to foreign keys. The infinity symbol indicates a many relationship. 


\section{DISCUSSION}

\section{Database Design}

Figure 1 shows the implementation of the database in Access with the tables and relationships. Central to the database is the Samples table. It is linked to the Gels and Separation tables. The Separation table contains the data on the preparative (chromatography) technique, and the Gels table contains data on the analytical (gel electrophoresis) technique. This arrangement allows both techniques to utilize the same samples and to maintain the links between the two.

There are several many-to-many relationships that require additional intersection tables. The relationship between Gels and Samples is a many to many, and the Wells table provides the link between them. The Wells table stores the data on the individual well loading (including a dilution factor). The relationship between the Separations table and Samples table requires the intersection table, Separation_Detail. The Separation_Detail table stores data on individual fractions (such as volume and time) and the identity of the sample applied to the chromatography (preparative) technique. The relationship between the Separations table and the Buffers table is also a many-tomany relationship (it allows the use of more than one buffer for many chromatography techniques). The Buffer detail table allows this relationship.

\section{Entering Data}

Samples can be categorized into stocks, fractions, or standards. The category is used for convenience to sort samples in these major groups. Standards imply that the samples are well characterized and used as a reference. Stocks indicate complex mixtures generally used as a starting material for a fractionation. Fractions indicate those samples that have been collected from a separation step. Data on the composition of a sample can be stored in the Components table (if desired).

There are three major forms that allow the user to add or edit data on gels, chromatography separations, and samples. Figure 2 shows the main form used to enter data on gels. There are combo boxes that allow the user to select the gel apparatus, the gel buffer type, and the staining technique. The combo boxes facilitate the entering of data into the forms. Data can be entered

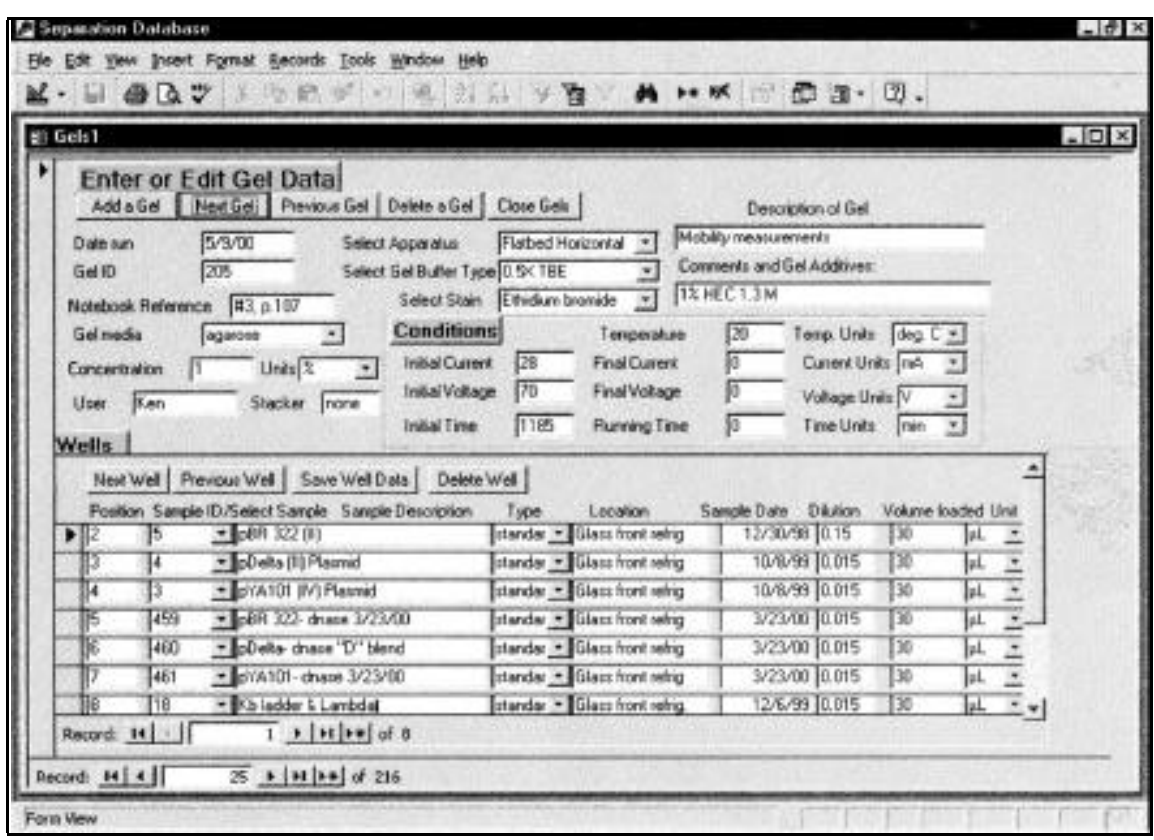

Figure 2. Major form to add or edit data associated with gel electrophoresis. The data associated with the analytical separation method are entered in the boxes or selected using the combo boxes. Sam ples can be selected from previously entered samples, or new samples can be entered directly from this form. The position, volume loaded, and dilution are specific to the gel separation. The data loaded on the sample description, type, location, and date are saved with the sample.

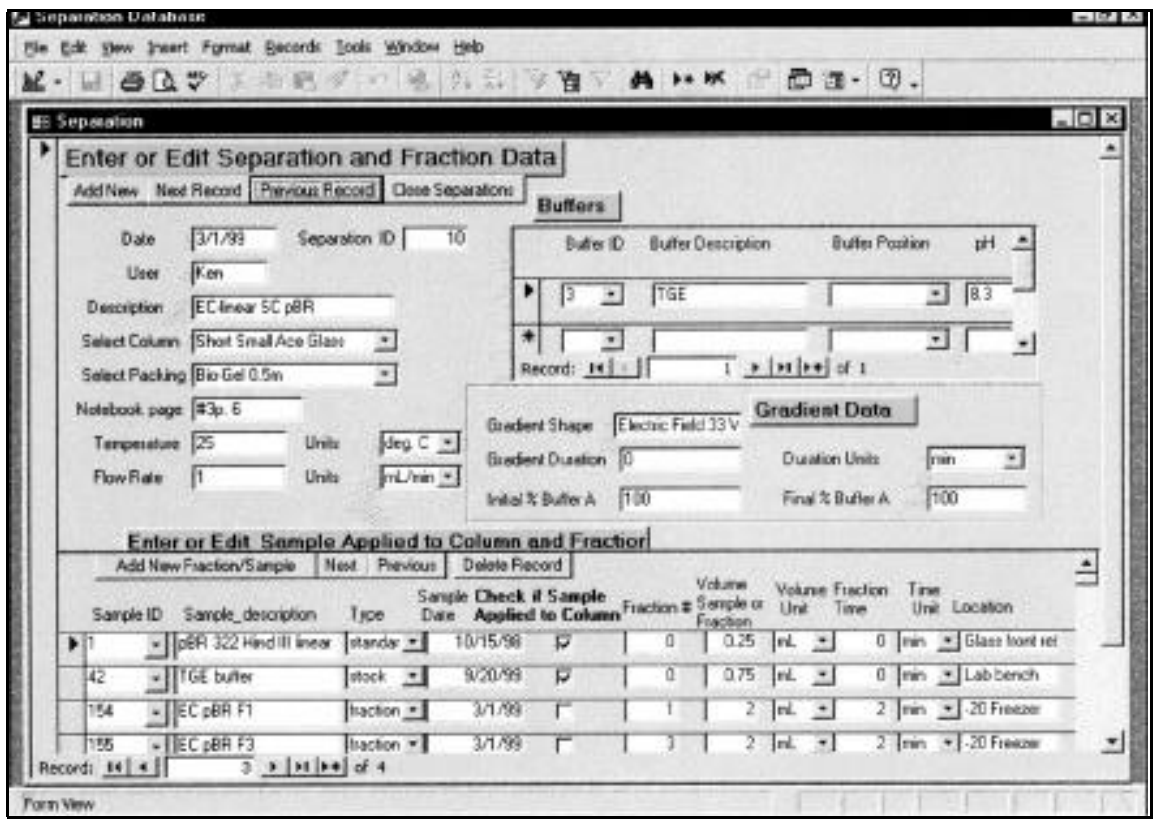

Figure 3. Major form to add or edit data associated with a chromatography technique. The data associated with the preparative separation method are entered in the boxes or selected using the combo boxes. Samples can be selected from previously entered samples, or new samples can be entered directly from this form. A checkbox indicates the sample was applied to the column. The fraction number, volume, time, and column sample selection are specific to the chromatography separation. The data loaded on the sample description, type, location, and date are saved with the sample. 
on the gel composition, electrophoresis conditions, and the well sample loading. Preexisting samples can be selected, or new samples can be added directly from this form.

A similar form (Figure 3) is used for adding data on the chromatography technique (the preparative separation). There are combo boxes that allow the user to select the column and packing materials (previously entered from their respective forms). A continuous subform is used to select the buffers used. Data are stored on the conditions used for flow and gradient conditions. A continuous subform is used to add and/or select the sample that is applied to the column (indicated by a checkbox). The sample applied to the column can be a preexisting sample, or a new sample can be added directly from this form. This form is also used to add the fractions and the fraction data (volume and times) that are associated with the particular separation. The samples (fractions) associated with a chromatography technique should be entered from this form (Separation) to maintain the link to the chromatography separation. Once the column sam ples and fractions are entered into the Samples table, they can be selected from the gel form when they are run on gels to be analyzed.

The Gels and Separation forms allow the user to add new samples directly from these forms as needed. Additional information on samples (such as standards) can be added from the Sam ples form. The Samples form allows additional data such as lot, concentration, and composition (if known). This form is mainly used for samples such as standards about which additional information is known.

Additional forms allow the user to add data on the apparatuses, buffers, gel types, staining, analysis, and the packing materials used. The data added in these forms allow the selection of these options from the combo boxes of the Gels and Separation forms.

\section{Retrieving Data}

There are continuous forms that allow the user to rapidly review the history of the gels, the column separations, and the samples stored in the database.
These forms allow the user to rapidly identify the particular gel, column separation, or sample to obtain a report. Clicking on a filter button allows the user to reduce the amount of displayed information. On the Gels and Separation forms, the user can filter for a user name to reduce the amount of data displayed. On the Samples form, the user can filter for the sample type (by entering standard, fraction, or stock) to reduce the amount of data displayed. After clicking on the report button, the user is prompted for an ID number for

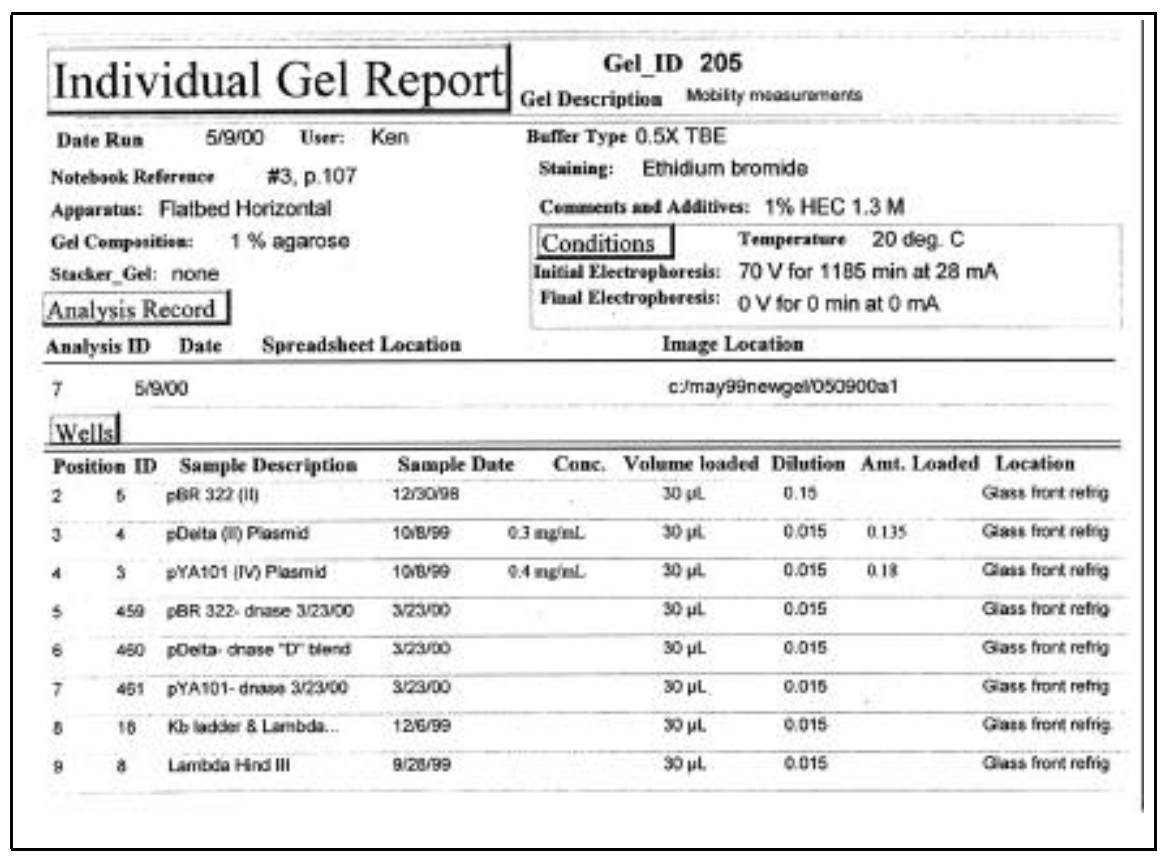

Figure 4. Report showing the data associated with a gel electrophoresis experiment. The report shows the separation conditions, the analysis history, and the sample data from a single gel.

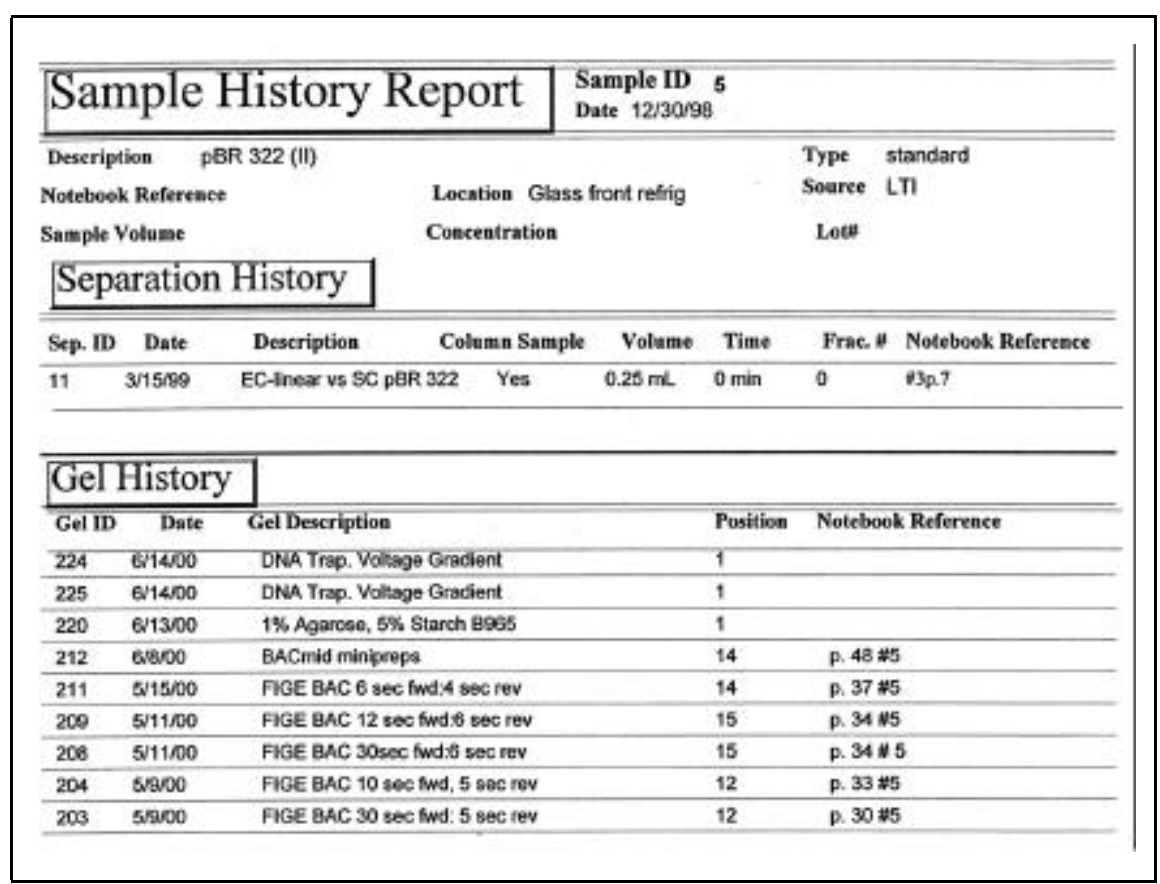

Figure 5. Report showing data associated with a sample separation history. The report shows both the analytical (gel electrophoresis) and preparative (chromatography) separation history of a selected sample. 
the gel, separation, or sample (depending on the form). A report will then be displayed containing the relevant data that can be printed. Figure 4 is an example of a gel data report. There are reports for chromatography conditions and sample histories (Figure 5).

There are two simple reports that can be exported into a Microsoft Excel $^{\circledR}$ spreadsheet (or other spreadsheet programs). The user opens the relevant spreadsheet report, selects the Save As/Export option from the file menu, and then saves to an external file (as an Excel file type or other format). This pastes the data from a gel or a separation (relating to samples and fractions) into the spreadsheet. The sample and fraction data can then be associated with data obtained from other sources, such as image analysis programs or detector outputs (for chromatographic fractions). We find that spreadsheets are a flexible and effective way to manipulate the data on the samples and fraction (from the separation database) with data from these other sources.

\section{SUMMARY}

This example database provides an effective way to store information on separation conditions and samples and to maintain the links between them. The database is capable of storing a large amount of data and retrieving it in a convenient manner. Currently, we have data from several hundred gels stored in the database. This database could be customized for the individual needs of other laboratories by adding or deleting fields. Many of the fields are optional and are only used when it is necessary to store that information on the separations or samples. Custom queries and reports can be generated to fit the needs of individual laboratories by consultation with texts on Access $(1,4)$.

Access gives a unique ID number to each sample, which will not be used again, even when a sample is deleted from the table. Users should be careful when changing data associated with a sample because these changes will be reflected in earlier records that have used that sample. The data that are part of a sample's attributes includes its description, type, concentration, location, date prepared, lot information, and components. Other data about the sample are associated with a sample through a gel or a chromatography step. Examples of data associated with a sample through a gel include well position, volume loaded, and dilution. Data associated with a sample through a separation include fraction volume, fraction time, fraction number, and column sample. It is important to understand this division of data to avoid changing the data that are part of a sample when changing the sample data associated with a particular separation.

One suggestion to make the organization of samples easier is to make copies of the database and give them different names for particular applications. For instance, DNA separations and protein separations are two applications that are unlikely to share the same samples. If the Samples table becomes too large to easily find samples, copies of the database should be made, and then samples should be deleted from the operating database. At a later time, the new data can be appended into a master database, if this is required.
The printed reports are a convenient way to store separation data in notebooks, and the electronic version provides a valuable backup that permits easy searches. Readers interested in obtaining a free copy of the database can contact the author by e-mail (kenneth. cole@nist.gov) or in writing.

\section{NOTE}

Certain commercial equipment, instruments or materials are identified in this paper to specify adequately the experimental procedure. Such identification does not imply recommendation by the National Institute of Standards and Technology, nor does it imply that the materials or equipment are necessarily the best available for the purpose.

\section{REFERENCES}

1.Gifford, D.R., D. Carollo, A. Feinberg, S. Forte, and J. Goodling. 1998. Access 97 Unleashed. Sams, Indianapolis, IN.

2.Nayler, O. and S. Stamm. 1999. ScienceLabDatabase: a computer program to organize a molecular biology laboratory. BioTechniques 26:1186-1191.

3.Ouchi, G.I. 1999. Getting started with database management system software. LC-GC 17:924-929.

4.Viescas, J. 1997. Running Microsoft Access 97. Microsoft Press, Redmond, WA.

Received 18 January 2000; accepted 22 August 2000.

Address correspondence to:

Dr. Kenneth D. Cole

Bioprocess Engineering Group, Stop 8312

Biotechnology Division

National Institute of Standards and Technology

Gaithersburg, MD 20899, USA.

e-mail:kenneth.cole@nist.gov 